The ASTrophysical Journal SUPPLEMENT SERIES, 90:807-811, 1994 February

(C) 1994. The American Astronomical Society. All rights reserved. Printed in U.S.A.

\title{
RADIO EMISSION FROM SNR 1987A
}

\author{
J. G. KIRK AND P. DUFFY \\ Max-Planck-Institut für Kernphysik, W-6900 Heidelberg, Germany \\ AND \\ LEWIS BALL \\ Research Centre for Theoretical Astrophysics, University of Sydney, N.S.W. 2006, Australia \\ Received 1993 March 3; accepted 1993 May 27
}

\begin{abstract}
Diffusive acceleration of electrons by the blast wave of SNR 1987A is shown to provide a reasonable fit to observations of the radio emission since it reappeared in 1990 July. As well as determining the diffusion coefficient in this model, the observations indicate that the compression ratio of the gas subshock embedded in the blast wave is $\sim 2.7$, much lower than the value of 4 expected of a strong shock front. We propose that protons accelerated by the shock front itself cause the weakening of the subshock by both heating and accelerating the upstream plasma, producing a structure with a precursor to the gas subshock. Preliminary calculations using a fluid model for the energetic particle population indicate that these effects can significantly weaken the subshock just a few years after the explosion, if the same rate of particle injection is assumed as in other models of the acceleration of cosmic rays.
\end{abstract}

Subject headings: acceleration of particles - ISM: individual (SNR 1987A) — radio continuum: ISM — shock waves

\section{INTRODUCTION}

Following a brief but powerful flare a few days after explosion, Supernova 1987 A disappeared from view as a radio source for $\sim 3$ years. However, it reappeared in July of 1990 and has displayed a monotonically increasing flux ever since (Staveley-Smith et al. 1992). In this paper we briefly review a model of the emission in which electrons are accelerated at the blast wave by the process of "diffusive shock acceleration" and subsequently emit synchrotron radiation in the magnetic field of the progenitor's stellar wind, which has been swept-up and compressed by the shock wave (Ball \& Kirk 1992). Using a simplified treatment of the time dependence of the acceleration process, and assuming constant speed and strength of the shock front, we derive the characteristic light curve expected when the rate at which electrons are injected into the acceleration process jumps from zero to a constant value. In this way, we attempt to model what might happen when the shock front encounters an irregularity-a "clump" of enhanced density or a zone of stronger than average turbulence-in the progenitor's wind. At any given frequency, this characteristic light curve rises rapidly at first, reaches a maximum and declines slowly. The decline comes about because the electrons suffer adiabatic losses as they stream outward with the shocked plasma after acceleration, and because we assume a $1 / r$ dependence of the magnetic field (where $r$ is the distance from the site of the explosion ). Comparing our theoretical light curves with observations we find a good fit is obtained if the supernova shock front encountered two "clumps" at different epochs.

\footnotetext{
${ }^{1}$ Invited contribution presented at IAU Colloquium 142, College Park, 1993 January.
}

The radio emission turned on first at low frequency ( 843 $\mathrm{MHz}$ ) and was detected at high frequency $(4.8 \mathrm{GHz})$ some 60 days later. Correspondingly, the initially steep spectrum $[\alpha>$ 1.6, for flux density $F(\nu) \propto \nu^{-\alpha}$ ] subsequently flattened, and settled down into a power law of index $\alpha=0.8-1$. Such a spectrum is compatible with diffusive acceleration at a shock front only if the compression ratio is $2.5-2.9$. This presents a problem, since the Rankin-Hugoniot conditions for a hydrodynamic shock front require a Mach number of less than 2 for such a low compression ratio. Because the supernova blast wave is still moving at high velocity $\left(\sim 30,000 \mathrm{~km} \mathrm{~s}^{-1}\right.$; Staveley-Smith et al. 1993), a Mach number of 2 requires an unrealistically high temperature in the ambient upstream plasma. We overcome this difficulty by assuming that the shock front accelerates not only electrons, but also protons. Two effects then occur: the upstream plasma is accelerated before reaching the shock discontinuity by the pressure gradient of energetic particles, and at the same time it is heated by the Alfvén wave turbulence which they induce. In $\S 3$ we present preliminary calculations of this effect using a two-fluid model similar to those used in studies of cosmic-ray acceleration in the later stages of SNR evolution (Duffy 1992; Kang \& Jones 1991).

\section{ELECTRON ACCELERATION}

The model for calculating the temporal and spatial dependence of the electron distribution and, from it, the synchrotron emission, is presented in detail by Ball \& Kirk (1992). It is based on a division of each emitting clump into two parts: an acceleration region close to the shock front and an expansion region downstream of it. We assume the electrons in the acceleration region behave as if they were encountering a plane shock. Once they escape into the expansion region, they cease to diffuse and remain frozen into the fluid flow, suffering only 
adiabatic losses. Consider a plane shock front of compression ratio $\rho=v_{1} / v_{2}$ into which plasma flows along the normal at speed $v_{1}$ and exits also along the normal at speed $v_{2}$. This shock accelerates electrons which diffuse in the background plasma with a spatial diffusion coefficient $\kappa$, and which enter the acceleration process at the rate $Q$ with momentum $p_{0}$. Although it is possible to write down the distribution function of such particles as a function of momentum, time, and space (Toptygin 1980; Drury 1991), we use a spatially averaged model (Axford 1981; Bogdan \& Völk 1983): an electron is presumed to undergo continuous acceleration while in the vicinity of the shock, such that its momentum $p$ increases at a rate $p \Delta / t_{c}$, where $\Delta=4\left(v_{1}-v_{2}\right) / 3 v$ is the average fractional momentum gain per shock crossing/recrossing (with $v$ the electron's velocity) and $t_{c}=4 \kappa\left(1 / v_{1}+1 / v_{2}\right) / v$ is the average time taken to perform such a cycle (Bell 1978; Drury 1983). In addition, these electrons escape from the shock region at a rate which is just the escape probability per cycle $P_{\text {esc }}=4 v_{2} / v$ divided by the average cycle time. Assuming $t_{c}$ independent of $p$, and time-independent injection for $t \geq t_{a}$, we find the synchrotron flux density emitted by a single clump is

$$
\begin{aligned}
F(\nu, t)=C(\hat{v} \hat{t})^{-\alpha}\{ & \left(\frac{\rho}{\rho-1}\right)^{\alpha}\left[B_{y_{1}}\left(\alpha, 1+\frac{4 \alpha}{3}\right)\right. \\
& \left.\left.\quad-B_{y_{2}}\left(\alpha, 1+\frac{4 \alpha}{3}\right)\right]+\frac{\eta}{2 \alpha \hat{t}} \rho^{-4 \alpha / 3}\right\},
\end{aligned}
$$

where $C$ is a constant, $\hat{t}=t / t_{0}$ is the dimensionless time, normalized to an arbitrary fiducial time $t_{0}, \hat{\nu}=\nu /\left(a_{1} B_{0} p_{0}^{2}\right)$ is the dimensionless frequency (with $a_{1}=1.3 \times 10^{10} \mathrm{~Hz} \mathrm{~T}^{-1}, B_{0}$ the magnetic field at $\hat{t}=1$ and $p_{0}$ the momentum with which electrons are injected), $\alpha=P_{\text {esc }} /(2 \Delta)$ and $B_{y}(a, b)$ is the incomplete $\beta$ function (Abramowitz \& Stegun 1972). The quantity $y_{1}=(\rho-1) \hat{t} /\left[\rho \hat{R}_{1}+(\rho-1) \hat{t}\right]$ is found by computing the root $\hat{R}=\hat{R}_{1}$ of the function

$$
\begin{aligned}
f(\hat{R})=\hat{\nu}(\rho \hat{R})^{-4 / 3}[\hat{R}+\hat{t}(\rho-1) / \rho]^{7 / 3} & \\
& -\exp \left[2\left(\hat{R}-\hat{t}_{a} / \rho\right) / \eta\right],
\end{aligned}
$$

and $y_{2}=(\rho-1) / \rho$. The small dimensionless parameter $\eta=$ $t_{c} /\left(\Delta \rho t_{0}\right)$ is roughly the acceleration timescale in units of $t_{0}$, and equation (1) is valid only when cut-offs in the electron distribution are unimportant, i.e., within the frequency range $\hat{t}^{-1}<\hat{\nu}<\hat{\nu}_{\max }=\hat{t}^{-1} \exp \left[2\left(\hat{t}-\hat{t}_{a}\right) / \rho \eta\right]$.

The basic features of the predicted emission from a single clump are illustrated in Figures 1 and 2, both plotted with $t_{0}, \rho$, $\eta$ and $\hat{t}_{a}$ chosen to fit the emission from the first clump encountered by the blast wave of SNR 1987A, as discussed below. A power law of index $\alpha$ is obtained extending from $\hat{v}=1 / \hat{t}$ up to close to $\hat{\nu}_{\max }$. The emission at a fixed frequency first rises as particles are accelerated up to the required energy, and then falls as adiabatic losses set in and the freshly injected particles, which feel a weaker magnetic field, are no longer able to compensate.

As discussed by Ball \& Kirk (1992) the observed time dependence of the flux from SNR 1987A at $843 \mathrm{MHz}$ and 4.8 $\mathrm{GHz}$ fits a model in which two clumps have been encountered by the shock front. This is illustrated in Figure 3.

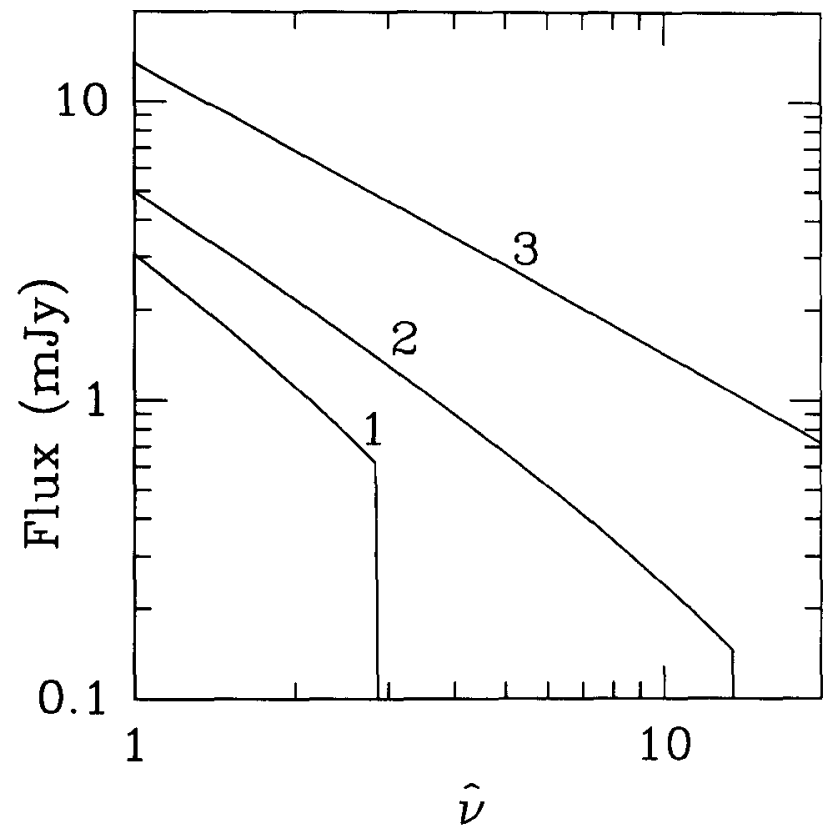

FIG. 1.-The predicted emission spectra from a single clump, at fixed times denoted by the labels 1: 1223 days, $2: 1260$ days, and 3: 1500 days.

However, the property of the emission of primary interest here is its spectrum. During the period when SNR 1987A was detectable at $843 \mathrm{MHz}$ and not at $4.8 \mathrm{GHz}$ the spectrum was steeper than $v^{-1.6}$. After switch on at $4.8 \mathrm{GHz}$ it quickly flattened from a power law of spectral index $\alpha \sim 1.3$ to one with $\alpha=0.8-1$. If the supernova blast wave is a simple sharp discontinuity in the velocity field as a function of radius, diffusive acceleration predicts a rapid flattening of the spectrum to a

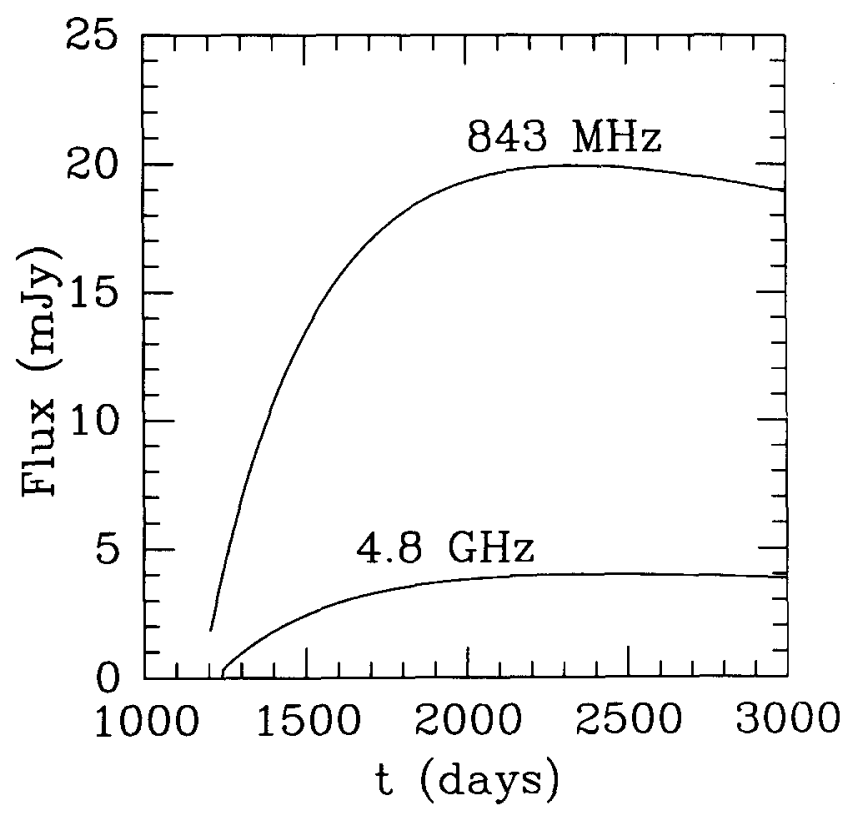

FIG. 2.-The predicted light curves at $843 \mathrm{MHz}$ and $4.8 \mathrm{GHz}$, from a single clump. 


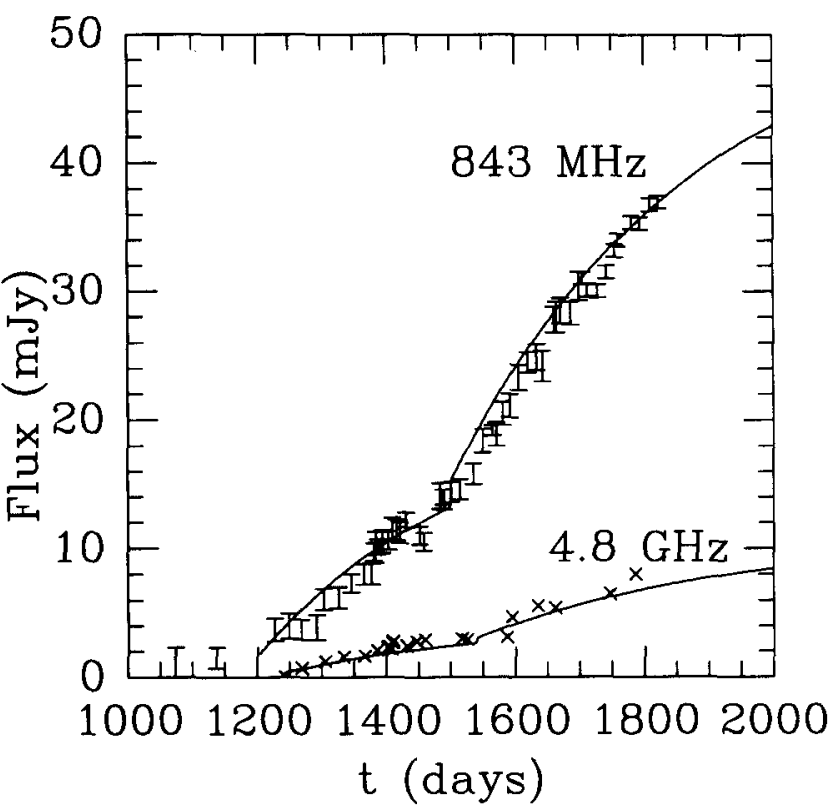

Fig. 3.-The predicted light curves $843 \mathrm{MHz}$ and $4.8 \mathrm{GHz}$, from two clumps of material, superposed on the observations. For clarity, only a subset of the data at $843 \mathrm{MHz}$ is shown. The errors on the $4.8 \mathrm{GHz}$ data are comparable to the size of the crosses.

value $\alpha=3 /[2(\rho-1)]$, and our best fit to the observations thus indicates $\rho=2.7$ rather than the value 4 expected of a strong shock front in a gas whose ratio of specific heats is $5 / 3$. Because the supernova remnant is still well within its free expansion phase the downstream plasma velocity is still roughly $30,000 \mathrm{~km} \mathrm{~s}^{-1}$. Compared to the undisturbed upstream plasma, this corresponds to a very high Mach number, so that the shock front in this simple picture is certainly strong.

It appears, therefore, that either the diffusive mechanism is not responsible for the radio emission, or that the picture of the blast wave as a simple discontinuity is incorrect.

\section{STRUCTURE OF THE BLAST WAVE}

There has been much interest recently in the problem of calculating the efficiency with which supernova remnants can accelerate cosmic rays (Drury, Markiewicz, \& Völk 1989; Kang \& Jones 1991; Duffy 1992). A useful approach is the "two-fluid" model, in which not only the background plasma, but also the accelerated particles (essentially protons) are described by fluid equations. Although open to criticism because of its crude parameterization of the injection process (Zank, Webb, \& Donohue 1992) and its circumvention of the closure problem (Jones \& Kang 1992; Völk et al. 1991) this method provides a relatively easy way of estimating whether or not the blast wave of SNR 1987A is capable of producing the observed steep spectrum synchrotron emission via diffusive acceleration of electrons.

In the free expansion phase, the material ejected in the supernova explosion expands at roughly constant velocity. To model this, we consider a spherically symmetric "piston" moving radially at constant speed into a medium containing an ideal gas of specific heat ratio $5 / 3$, and a gas of energetic particles (protons) with specific heat ratio $4 / 3$ and an effective spatial diffusion coefficient $\bar{\kappa}$. Assuming velocity gradients in the shocked gas remain small, the piston motion is implemented by requiring that the plasma speed immediately downstream of the gas subshock is constant. Starting a few days after explosion, we allow a small amount of energy to be injected into the protons by the shock front, in the same way as described by Duffy (1992). We then solve numerically the coupled fluid equations to find the structure of the precursor built up by the protons as they are accelerated by the shock front. Two effects are important. First, the pressure gradient of cosmic rays acts on the upstream plasma and accelerates it radially outward before it encounters the subshock, reducing the speed with which plasma flows into the subshock. Second, the Alfvén waves which are responsible for scattering the energetic particles do work on the plasma and, therefore, heat it. To model this effect, which reduces the Mach number of the subshock, we use the equations given by McKenzie \& Völk (1982) (see also Völk, Drury, \& McKenzie 1984) and assume the energy put into Alfvén wave turbulence by the cosmic-ray streaming instability is immediately and locally absorbed by the background plasma. The entropy production rate in the background plasma is then simply proportional to the Alfvén speed and the cosmic-ray pressure gradient:

$$
T \frac{d S}{d t}=v_{\mathrm{A}} \frac{d P_{c}}{d r}
$$

where $T$ is the plasma temperature and $S$ the entropy density. The results of a typical run are shown in Figures 4 and 5. We choose a proton injection rate such that five particles in 1000 incident on the subshock is injected with twice the thermal energy of the downstream plasma-a value similar to those used by Drury, Markiewicz, \& Völk (1989). The velocity of postshock plasma was fixed to $2.25 \times 10^{7} \mathrm{~m} \mathrm{~s}^{-1}$ in order to give an initial shock speed of $30,000 \mathrm{~km} \mathrm{~s}^{-1}$, the density, temperature and magnetic field of the upstream plasma were taken according to the estimates of Kirk \& Wassmann (1992) and the average proton diffusion coefficient, $\vec{k}$, was assumed to be a fraction $\left(\frac{1}{5}\right)$ of the Bohm value appropriate to protons of momentum $p_{\max }$. The time dependence of the subshock compression ratio $(\rho)$, the temperatures (in Kelvins) immediately upstream and downstream of the subshock, the small parameter $\bar{\kappa} /\left(R_{s} v_{s}\right)$ (where $R_{s}$ is the radius of the subshock and $v_{s}$ is the speed of the subshock in the rest frame of the progenitor), and the maximum momentum $p_{\max }$ are displayed in the four plots of Figure 4. Here the abscissa is time since explosion (in years),

Figure 5 shows the radial dependence of velocity (in $\mathrm{m} \mathrm{s}^{-1}$ ), density (in $\mathrm{kg} \mathrm{m}^{-3}$ ), and gas and cosmic-ray pressures (in $P$ ) as they appear 4 years after explosion. According to Ball \& Kirk (1992) the electrons responsible for the radio emission have a diffusion coefficient $\kappa \approx 2 \times 10^{20} \mathrm{~m}^{2} \mathrm{~s}^{-1}$. The distance which they can penetrate into the precursor from the shock front can be estimated as $\kappa / v_{s} \approx 10^{13} \mathrm{~m}$, and is, therefore, very small compared to the size of the precursor, which is of the order of $10^{15} \mathrm{~m}$. Consequently, if these electrons are accelerated in a blast wave similar to that of Figure 5, their spectrum will reflect the compression ratio across the subshock, not across the entire structure. Figure 4 shows that this quantity $(\rho)$ drops to a 
(a)

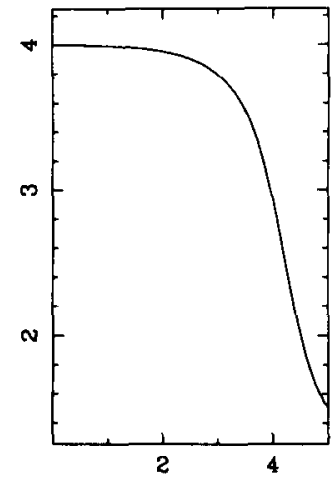

time (in years)

(c)

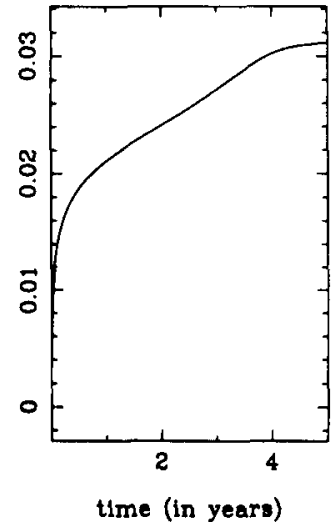

FiG. 4.-The time dependence of $(a)$ the subshock compression ratio, $(b)$ the pre- and postshock temperatures (in Kelvins), $(c)$ the small parameter $\bar{\kappa} /\left(R_{s} v_{s}\right)$, and $(d)$ the logarithm of the maximum proton momentum (in units of the rest mass times the speed of light).

value compatible with the observed synchrotron spectrum after $\sim 3$ years. It is interesting to note that Ellison \& Reynolds (1991) have used an argument similar to this to explain the steep spectra observed in older supernova remnants. Their treatment, however, relies on the blast wave structure being stationary, whereas our calculations follow the evolution in the early free expansion phase.

\section{DISCUSSION}

One of the main problems confronting the hypothesis that diffusive acceleration of electrons is responsible for the radio emission of SNR 1987A is the steepness of the observed spectrum. We have presented preliminary calculations which indicate that the steepening could be a result of the back-reaction of cosmic rays (other than electrons) which have also been (a)

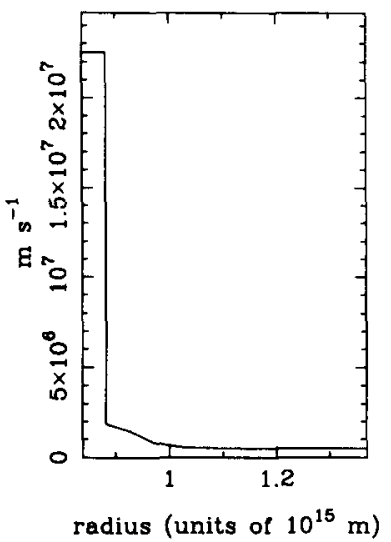

(c)

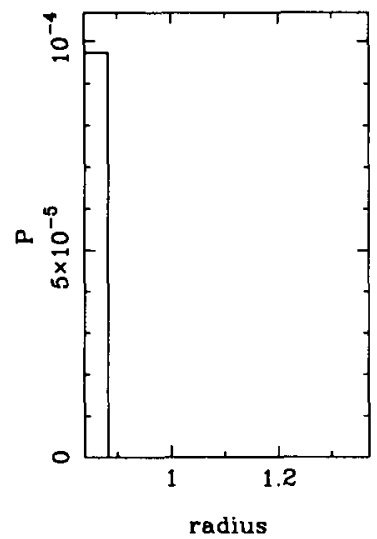

(b)

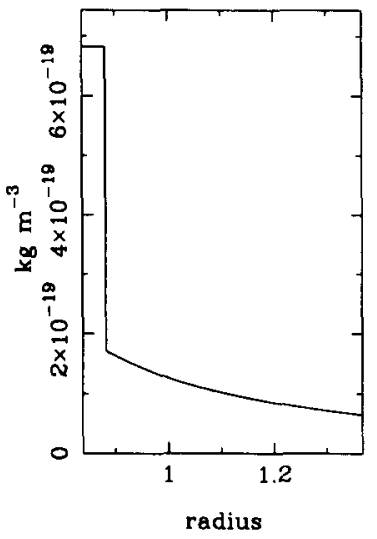

(d)

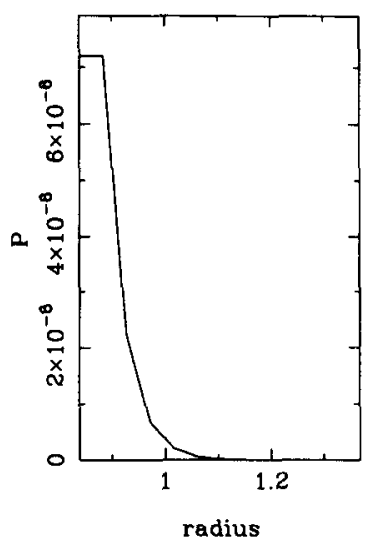

FIG. 5.-The space dependence of $(a)$ the velocity, $(b)$ density, $(c)$ gas pressure, and $(d)$ cosmic-ray pressure in the precursor to the shock, after 4 years.

accelerated by the shock. In fact, when we keep the parameter used to control injection constant in time, our computations show the gas subshock weakens and the system becomes dominated by the pressure of energetic protons after only 5 years. This result is potentially important not only for theories of the radio emission from SNR 1987A, but also for theories of the origin of cosmic rays. It raises the possibility that the early evolution of a supernova remnant could depend sensitively on the level of injection of protons. However, our results are preliminary and it would be desirable to perform calculations with better spatial resolution of the precursor. Clearly, further work is required on the time-dependent acceleration of both the proton and electron components of the energetic particle population, as well as on the problem of injection.

\section{REFERENCES}

Abramowitz, M., \& Stegun, I. E. 1972, Handbook of Mathematical Functions (Washington, DC: NBS)

Axford, W. I. 1981, Proc. 17th. Internat. Cosmic Ray Conf. (Paris), 12, 155

Ball, L. T., \& Kirk, J. G. 1992, ApJ, 396, L42

Bell, A. R. 1978, MNRAS, 182, 147
Bogdan, T. J., \& Völk, H. J. 1983, A\&A, 122, 129

Drury, L. O'C. 1983, Rep. Progr. Phys., 46, 973

. 1991, MNRAS, 251, 340

Drury, L. O'C., Markiewicz, W. J., \& Völk, H. J. 1989, A\&A, 225, 179

Duffy, P. 1992, A\&A, 262, 281

Ellison, D. C., \& Reynolds, S. P. 1991, ApJ, 382, 242 
Jones, T, W., \& Kang, H. 1992, ApJ, 396, 575

Kang, H., \& Jones, T. W. 1991, MNRAS, 249, 439

Kirk, J. G., \& Wassmann, M. 1992, A\&A, 254, 167

McKenzie, J. F., \& Völk, H. J. 1982, A\&A, 116, 191

Staveley-Smith, L., et al. 1992, Nature, 355, 147

1993, Proc. Astron. Soc. Australia in press

Toptygin, I. N. 1980, Space Sci. Rev., 26, 157
Turtle, A. J., et al. 1987, Nature, 327, 38

Völk, H. J., Drury, L. O'C., Duffy, P., \& Markiewicz, W. J. 1991, Proc. 22d Internat. Cosmic Ray Conf. (Dublin), 2, 205

Völk, H. J., Drury, L. O'C., \& McKenzie, J. F. 1984, A\&A, 130, 19

Zank, G. P., Webb, G. M., Donohue, D. J. 1992, in AIP Conf. Proc. 264, Particle Acceleration in Cosmic Plasmas, ed. G. P. Zank \& T. K. Gaisser (New York: AIP) 158 